\title{
Application of Ultrasound-guided Core Needle Biopsy in the Diagnosis of T3 or T4 Stage Laryngeal and Hypopharyngeal Cancer
}

\author{
YUNQIANG WANG ${ }^{1 *}$, DAJIAN LI ${ }^{2 *}$, RUIHUA LIU ${ }^{3 *}$, CHUANLIANG JIA $^{2}$, AIHUA JIANG ${ }^{4}$, \\ YAYUN LV ${ }^{5}$, SHUFEN XU ${ }^{2}$, HUA ZHANG ${ }^{2}$, XIAOLI CAO ${ }^{3}$ and XICHENG SONG ${ }^{2}$ \\ Departments of ${ }^{1}$ Imaging of Head and Neck, ${ }^{2}$ Otorhinolaryngology and Head and Neck Surgery, \\ ${ }^{3}$ Ultrasonography and ${ }^{4}$ Anesthesia, Yuhuangding Hospital of Qingdao University, Yantai, P.R. China; \\ ${ }^{5}$ Binzhou Medical University, Yantai, P.R. China
}

\begin{abstract}
Aim: To study the value of ultrasound-guided core needle biopsy $(\mathrm{CNB})$ in the diagnosis of T3 or T4 stage laryngeal and hypopharyngeal cancer, which is difficult by routine methods. Patients and Methods: Nineteen cases of T3 or T4 stage laryngeal or hypopharyngeal carcinoma with abnormal pharyngeal sensitivity, severe dyspnea, submисои cancer recurrence, cardiovascular and pulmonary dysfunction were reviewed retrospectively from October 2012 to October 2014 in the Yuhuangding Hospital of Qingdao University. Ultrasound-guided coarse needle biopsies were used on primary lesions after assessing the patients with neckenhanced computed tomography $(C T)$ or magnetic resonance imaging (MRI) scan(s). The clinical value of ultrasoundguided CNB in the diagnosis of laryngeal and hypopharyngeal cancer was analyzed. Results: All patients underwent successful pathological diagnosis by ultrasound-guided CNB without any serious complications. Dyspnea, cardiovascular and pulmonary dysfunction did not deteriorate. Conclusion: Ultrasound-guided CNB is a highly safe and efficient method for the pathological diagnosis of T3 or T4 stage laryngeal and hypopharyngeal cancer. It should be used especially when the fiberoptic or laryngoscope biopsy are of high risk.
\end{abstract}

High-frequency ultrasonography is routinely used as an imaging method to evaluate the involvement of cervical

*These Authors contributed equally to this study.

Correspondence to: Xicheng Song, MD, Ph.D., Department of Otorhinolaryngology and Head and Neck Surgery, Yuhuangding Hospital of Qingdao University No 20, East Yuhuangding Road, Yantai 264000, P.R. China. Tel: +86 05356691999-83523 Fax: +86 05356240341, e-mail: songxicheng@126.com

Key Words: Laryngeal cancer, hypopharyngeal cancer, biopsy, core needle, ultrasound. lymph nodes, soft tissues and thyroid gland in patients with laryngeal or hypopharyngeal carcinoma, but rarely to evaluate the tumor itself, mainly because of the calcified thyroid cartilages in adults and the interference of air within the laryngeal cavities. Nevertheless, attempts have been made to use ultrasonography in this area, largely to take advantage of its non-invasive and real-time imaging features. Since the 1970's, ultrasonography has been used to evaluate laryngeal tumors in a few studies, with some of these proving that ultrasonography may assist tumor staging in patients with advanced laryngeal cancer (1).

Core needle biopsy (CNB) has been proposed as an additional diagnostic method to ultrasound-guided fine needle aspiration biopsy (US-FNA), mainly to overcome the limitations of inconclusive cytologic diagnosis. CNB provides a large amount of tissue that enables histological diagnosis and additional immunohistochemical staining, if needed. Several studies have shown the usefulness of CNB in providing definitive diagnosis for thyroid nodules (2-5).

Therefore, for the above two reasons, ultrasound-guided $\mathrm{CNB}$ in the diagnosis of laryngeal or hypopharyngeal cancer, especially in T3 or T4 stage, is, theoretically, feasible. We applied this technique in the clinic to patients who had difficulties in pathological diagnosis by conventional biopsy for reasons, such as abnormal pharyngeal sensitivity, severe dyspnea, submucous cancer recurrence, cardiovascular and pulmonary dysfunction.

\section{Patients and Methods}

Patients and clinical data. Nineteen cases of T3 or T4 stage laryngeal or hypopharyngeal carcinoma with abnormal pharyngeal sensitivity, severe dyspnea, submucous cancer recurrence, cardiovascular and pulmonary dysfunction were reviewed retrospectively from October 2012 to October 2014 in Yuhuangding Hospital of Qingdao University. Of these patients, all were males aged 60-92 years (mean=70.53), 4 cases had abnormal pharyngeal 
Table I. Patients and clinical data.

\begin{tabular}{|c|c|c|c|c|}
\hline Age & Lesion site & Risk factors & Pathologic diagnosis & T stage \\
\hline 60 & Glottic region & Abnormal pharyngeal sensitivity, severe dyspnea & Squamous cell carcinoma & $\mathrm{T} 3$ \\
\hline 63 & Glottic region & Abnormal pharyngeal sensitivity, severe dyspnea & Squamous cell carcinoma & $\mathrm{T} 3$ \\
\hline 65 & Glottic region & Ischemic heart disease, severe dyspnea & Squamous cell carcinoma & $\mathrm{T} 3$ \\
\hline 70 & Glottic region & Submucous cancer recurrence & Squamous cell carcinoma recurrence & $\mathrm{T} 3$ \\
\hline 71 & Glottic region & Coronary artery disease, severe dyspnea & Squamous cell carcinoma recurrence & $\mathrm{T} 4$ \\
\hline 82 & Supraglottic region & Ischemic encephalopathy & Squamous cell carcinoma & T3 \\
\hline 66 & Supraglottic region & Abnormal pharyngeal sensitivity, severe dyspnea & Squamous cell carcinoma & $\mathrm{T} 3$ \\
\hline 69 & Supraglottic region & Abnormal pharyngeal sensitivity, severe dyspnea & Squamous cell carcinoma & $\mathrm{T} 3$ \\
\hline 70 & Supraglottic region & Submucous cancer recurrence & Squamous cell carcinoma recurrence & $\mathrm{T} 4$ \\
\hline 63 & Pyriform fossa & Cerebral infarction sequelae & Squamous cell carcinoma & $\mathrm{T} 3$ \\
\hline 74 & Pyriform fossa & Coronary artery disease, severe dyspnea & Squamous cell carcinoma & $\mathrm{T} 3$ \\
\hline 66 & Pyriform fossa & Coronary artery disease, severe dyspnea & Squamous cell carcinoma & $\mathrm{T} 3$ \\
\hline 83 & Pyriform fossa & Coronary artery disease, severe dyspnea & Squamous cell carcinoma & $\mathrm{T} 3$ \\
\hline 92 & Pyriform fossa & Severe dyspnea & Squamous cell carcinoma & $\mathrm{T} 4$ \\
\hline 63 & Pyriform fossa & Severe dyspnea & Squamous cell carcinoma & $\mathrm{T} 4$ \\
\hline 61 & Epiglottis & Submucous cancer recurrence & Squamous cell carcinoma recurrence & $\mathrm{T} 4$ \\
\hline 80 & Aryepiglottic folds & Coronary artery disease, severe dyspnea & Squamous cell carcinoma & $\mathrm{T} 3$ \\
\hline 74 & Epiglottis & Chronic obstructive pulmonary disease, severe dyspnea & Squamous cell carcinoma & $\mathrm{T} 3$ \\
\hline 68 & Epiglottis & Coronary artery disease, severe dyspnea & Squamous cell carcinoma & $\mathrm{T} 3$ \\
\hline
\end{tabular}

sensitivity and I to II degree of laryngeal obstruction, 9 cases had severe cardiovascular-pulmonary dysfunction and I to II degree of laryngeal obstruction, 3 cases had submucous cancer recurrence, 3 cases had II degree of laryngeal obstruction (Table I). They all underwent a contrast-enhanced computed tomography (CT) and/or magnetic resonance imaging (MRI) examination prior to the ultrasound-guided CNB. Tumor-node-metastasis (TNM) staging was analyzed according to the standard of the International Union against Cancer (IUCC) in 2002. The clinical value of ultrasoundguided $\mathrm{CNB}$ in the diagnosis of laryngeal and hypopharyngeal cancer was analyzed by the clinical outcome.

Ultrasound-guided CNB procedure. We used an American Logiq E9 GE color ultrasonic diagnostic instrument (GE healthcare China, Wuxi, China), a United States Bard automatic ejection biopsy gun and the special matching $18 \mathrm{G}$ groove type tissue cutting needle (C. R. Bard, Inc., Murray Hill, NJ, USA). Carefully reading the CT and/or MRI data before puncture was necessary to determine the location of the tumor and surrounding tissue involvement (Figures 1 and 2). The location, size, shape, internal echo, boundary and internal blood flow distribution of the tumors had to be evaluated again by ultrasonic examination. Ultrasound imaging can display the vascular and blood flow area well in the neck. This helps the doctor choose the best puncture site and direction. We chose to puncture at the nearest skin site to the tumor and avoid the abundant blood circulation area and large blood vessels (Figures 3 and 4).

The patients were in the supine position, head backward (not too backward, avoiding the aggravation of laryngeal obstruction), with the anterior region of the neck being exposed. The puncture point was located and the depth of inserting was measured using an American Logiq E9 GE color ultrasonic diagnostic instrument. The skin of the anterior neck was sterilized with $0.5 \%$ iodophor. The puncture point was locally anesthetized with $4 \mathrm{mg} / \mathrm{kg} 1 \%$ lidocaine.
The biopsy needle punctured into different tumor areas, 2-3 times, along the long axis of the ultrasonic probe under the real-time ultrasonic monitoring (Figure 5) allowing for the collection of some pathological tissue (Figure 6). The pathological tissue was fixed with $4 \%$ formalin solution for further histological diagnosis and additional immunohistochemical staining examination.

\section{Results}

Of the patients involved in the study, 13 cases were laryngeal carcinoma, 6 cases hypopharyngeal carcinoma, 14 cases were in the T3 stage and 5 cases in the T4 stage. All patients had successful pathological diagnosis by only using ultrasound-guided CNB without any serious complications. Dyspnea, cardiovascular and pulmonary dysfunction did not deteriorate. All 19 cases were pathologically diagnosed as squamous cell carcinomas.

\section{Discussion}

Due to the calcified thyroid cartilage in adults and the interference of air within the laryngeal cavities, ultrasonography is rarely used to evaluate laryngeal or hypopharyngeal carcinoma structure(s). Nevertheless, attempts have been made to use ultrasonography in this area. Ultrasonography has been used to evaluate laryngeal tumors in a few studies since the 1970's and some of these have proved that ultrasonography can assist in tumor staging in patients with advanced laryngeal cancer (1, 6-8). With the application of a wide band and high frequency ultrasonic 


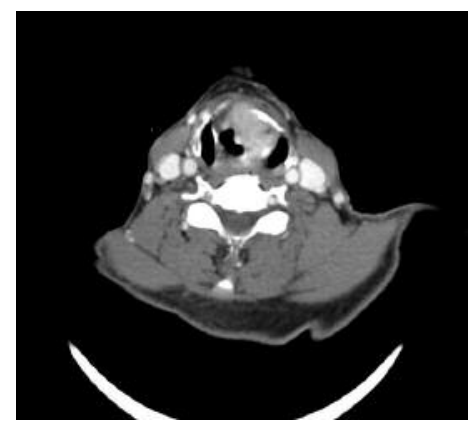

Figure 1. Enhanced computed tomography (CT) scan shows left laryngeal space and thyroid cartilage destruction.

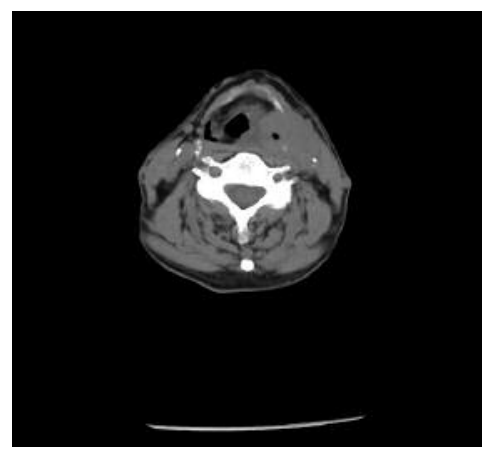

Figure 2. Computed tomography (CT) scan shows left pharyngeal space and partial thyroid cartilage destruction.

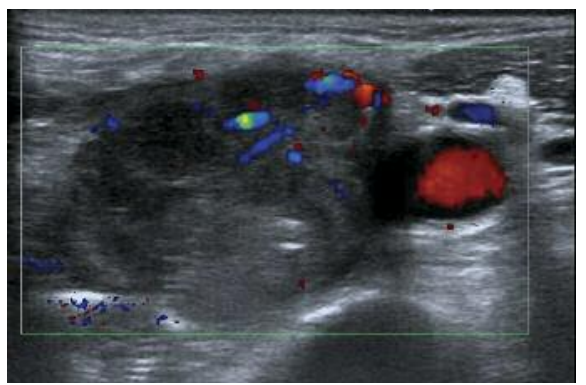

Figure 3. Ultrasound color imaging displays tumor and blood circulation.

probe, ultrasound can clearly show the main structures of laryngeal cartilage, ligament, muscle, pre-epiglottic space and para-glottic space for their superficial location and, to a certain extent, the sound transmission of laryngeal cartilage. The occupying solid tumor displaces the throat cavity and infiltrates and destroys the thyroid cartilage. It provides a favorable condition for ultrasound imaging $(6,7)$. The high resolution ultrasound for laryngeal imaging has the

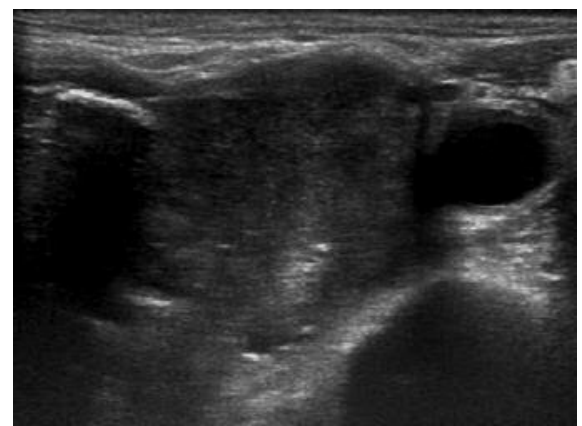

Figure 4. Gray-scale ultrasound imaging shows tumor adjacent to a cervical blood vessel.

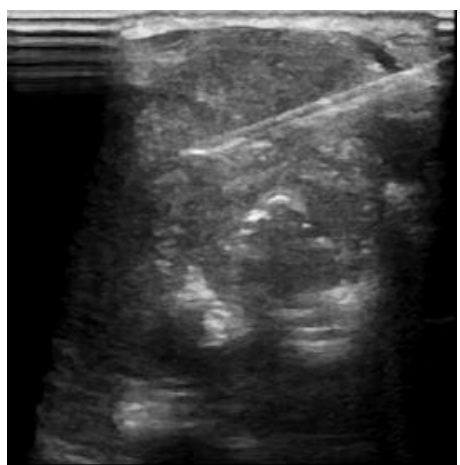

Figure 5. Ultrasound-guided needle biopsy of the solid part of the tumor.

advantage of real-time, dynamic, multi-plane, as well as multi-directional, imaging, which limits MRI and CT imaging (8). A T3 or T4 stage laryngeal and hypopharyngeal carcinoma is usually larger and has a wide range of infiltration. This, therefore, not only makes CNB under ultrasound guidance possible but also makes this method of very high clinical value.

At present, the fiber-optic and suspension laryngoscope biopsy are the two most common methods used to obtain histopathological tissue for laryngeal and hypopharyngeal carcinoma. However, these two kinds of methods are poorly tolerated by patients with abnormal pharyngeal sensitivity, severe dyspnea, submucous cancer recurrence, cardiovascular and pulmonary dysfunction. For the T3 or T4 stage laryngeal and hypopharyngeal cancer, most patients are elderly. In clinic, it is not unusual for the above criteria to be found together. Ultrasound-guided CNB can not only avoid the significant interference of the airway and cardiopulmonary function affecting the fiber-optic and supporting laryngoscopy biopsy but also has minimally invasive characteristics. It has an unparalleled advantage for the submucous recurrent carcinoma, which is highly difficult to sample using the fiber- 


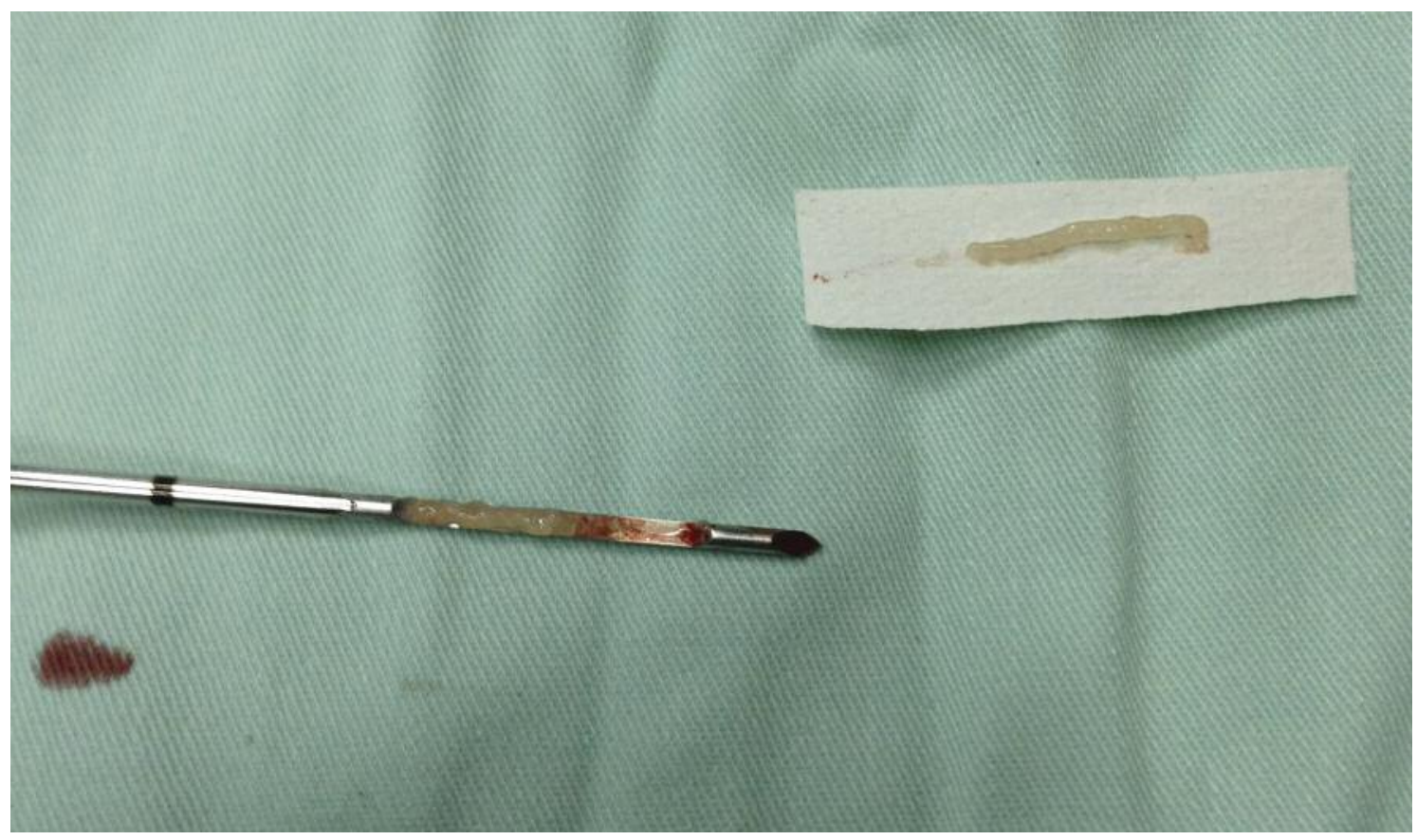

Figure 6. Core biopsy needle with pathological tissue.

optic and suspension laryngoscope biopsy. Imaging examination plays a very important role in the diagnosis of the recurrence of laryngeal and hypolaryngeal carcinoma; however, the final diagnosis still relies on pathological biopsy. Early surgery and radiotherapy result in extensive cervical edema, fibrosis, stiffness and stenosis of the larynx and trachea, which makes routine biopsy almost impossible. In this study, there were 3 cases of suspected recurrent cancer whose fiber-optic or supporting laryngoscopy biopsy showed negative results. We applied ultrasound-guided CNB and, finally, diagnosed submucosal squamous cell carcinoma recurrence. This approach provided an important basis for the next step of treatment options.

Ultrasound imaging can display the vascular and blood flow area well in the neck. This helps the doctor choose the best puncture site and direction and, effectively, avoid accidental injury. There were no serious complications for all patients in our study. Eight patients complained about mild pain in the puncture site, though this pain subsided within two days without the prescription of painkillers. Even so, it is necessary to carry out this operation to be performed by a senior ultrasonic doctor.

US-FNA is widely used in the pathological diagnosis of neck tumors, especially in thyroid tumors (9). The main difference between the fine needle biopsy and the coarse needle biopsy is the diameter of the cutting section. The material from the fine needle biopsy is enough for cytology examination, but not enough for immunohistochemical tests. CNB, on the other hand, is able to obtain adequate tissue to make up for this defect of the fine needle biopsy $(2,4,10-12)$.

Ultrasound examination is performed in real-time and is dynamic. The operator can observe the position of puncture needle throughout the whole procedure. It has been reported that CT-guided biopsy achieves satisfactory results (13). However, it is not real-time dynamic monitoring and patients have to receive more $\mathrm{X}$-ray irradiation $(1,7,14-16)$. Some have reported that three-dimensional Doppler ultrasound imaging has a high diagnostic value for laryngeal and hypopharyngeal cancer. However, the operation technique and image recognition still need to be improved $(17,18)$.

Ultrasound examination of laryngeal and pharyngeal cancer has certain limitations. Laryngeal cartilage calcification can reduce the sonolucency and affect the imaging of the posterior tumors. In order to show the panorama of the tumor, the cartilage between the ligaments has to be taken as an acoustic window to scan the tumor. It is not as accurate as the fiber laryngoscope to diagnose the ulcerative and early tumors. The air in the laryngeal cavity affects both the display of the posterior structure and color Doppler flow imaging by producing color flicker artifacts. The coronal scan should be done on both sides of the throat in order to reduce the influence of air interference. In this regard, it is necessary to strictly choose and evaluate patients. 


\section{Acknowledgements}

This study was financially supported by Yantai Science and Technology Planning Project 2016WS010.

\section{References}

1 Hu Q, Luo F, Zhu SY, Zhang Z, Mao YP and Hui Guan X: Staging of laryngeal carcinoma: comparison of high-frequency sonography and contrast-enhanced computed tomography. Clin Radiol 67: 140-147, 2012.

2 Na DG, Kim JH, Sung JY, Baek JH, Jung KC, Lee H and Yoo $\mathrm{H}$ : Core-needle biopsy is more useful than repeat fine-needle aspiration in thyroid nodules read as nondiagnostic or atypia of undetermined significance by the Bethesda system for reporting thyroid cytopathology. Thyroid 22: 468-475, 2012.

3 Choi SH, Baek JH, Lee JH, Choi YJ, Hong MJ, Song DE, Kim JK, Yoon JH and Kim WB: Thyroid nodules with initially non diagnostic, fine-needle aspiration results: Comparison of coreneedle biopsy and repeated fine-needle aspiration. Eur Radiol 24: 2819-2826, 2014

4 Sung JY, Na DG, Kim KS, Yoo H, Lee H, Kim JH and Baek JH: Diagnostic accuracy of fine-needle aspiration versus core-needle biopsy for the diagnosis of thyroid malignancy in a clinical cohort. Eur Radiol 22: 1564-1572, 2012.

5 Yeon JS, Baek JH, Lim HK, Ha EJ, Kim JK, Song DE, Kim TY and Lee JH: Thyroid nodules with initially nondiagnostic cytologic results: The role of core-needle biopsy. Radiology 268: 274-280, 2013.

6 Ying M, Bhatia K S, Lee Y P, Yuen HY and Ahuja AT: Review of ultrasonography of malignant neck nodes: Greyscale, Doppler, contrast enhancement and elastography. Cancer imaging 13(4): 658-669, 2012.

7 Hong SF, Lai YS and Lee KW: Efficiency of three-dimensional Doppler ultrasonography in assessing nodal metastasis of head and neck cancer. Eur Arch Otorhinolaryngol 272(10): 29852991, 2015.

8 Xia C X, Zhu Q, Zhao HX, Yan F, Li SL and Zhang SM: Usefulness of ultrasonography in assessment of laryngeal carcinoma. Br J Radiol 86(1030): 20130343, 2013.

9 Cooper DS, Doherty GM, Haugen BR, Kloos RT, Lee SL, Mandel SJ, Mazzaferri EL, McIver B, Pacini F, Schlumberger M, Sherman SI, Steward DL and Tuttle RM: Revised American Thyroid Association management guidelines for patients with thyroid nodules and differentiated thyroid cancer: The American Thyroid Association (ATA) guidelines taskforce on thyroid nodules and differentiated thyroid cancer. Thyroid 19(11): 11671214, 2009.

10 Min HS, Kim JH, Ryoo I, Jung SL and Jung CK: The role of core needle biopsy in the preoperative diagnosis of follicular neoplasm of the thyroid. APMIS 122: 993-1000, 2014.
11 Hahn SY, Shin JH, Han BK, Ko EY and Ko ES: Ultrasonography-guided core needle biopsy for the thyroid nodule: Does the procedure hold any benefit for the diagnosis when fine-needle aspiration cytology analysis shows inconclusive results? Br J Radiol 86(1025): 20130007, 2013.

$12 \mathrm{Li} \mathrm{L}$, Chen BD, Zhu HF, Wu S, Wei D, Zhang JQ and Yu L: Comparison of pre-operation diagnosis of thyroid cancer with fine-needle aspiration and core-needle biopsy: A meta-analysis. Asian Pac J Cancer Prev 15: 7187-7193, 2014.

13 Rousset J, Abgral R, Chinellato S, Garetier M, Barberot C, Valette G, Potard G, Le Bivic T and Salaun PY: Early recurrence or submucosal residual of laryngeal squamous cell carcinoma: Diagnosis by CT-guided endolaryngeal core biopsy on a transcutaneous approach. Head Neck 35(7): E202-204, 2013.

14 Schoellnast H, Komatz G, Bisail H, Talakic E, Fauster M, Ehammer T, Quehenberger F and Schaffler GJ: CT-guided biopsy of lesions of the lung, liver, pancreas or of enlarged lymph nodes: Value of additional fine needle aspiration (FNA) to core needle biopsy (CNB) in an offsite pathologist setting. Acad Radiol 17: 1275-1281, 2010.

15 Loh SE, Wu DD, Venkatesh SK, Ong CK, Liu E, Seto KY, Gopinathan A and Tan LK: CT-guided thoracic biopsy: Evaluating diagnostic yield and complications. Ann Acad Med Singapore 42: 285-290, 2013.

16 Lim LG, Lakhtakia S, Ang TL, Vu CK, Dy F, Chong VH, Khor CJ, Lim WC, Doshi BK, Varadarajulu S, Yasuda K, Wong JY, Chan YH, Nga ME and Ho KY: Factors determining diagnostic yield of endoscopic ultrasound guided fine-needle aspiration for pancreatic cystic lesions: A multicentre Asian study. Dig Dis Sci 58: 1751-1757, 2013.

17 Liberty G, Boldes R, Shen O, Shaul C, Cohen SM and Yagel S: The fetal larynx and pharynx: Structure and development on two- and three-dimensional ultrasound. Ultrasound Obstet Gynecol 42(2): 140-148, 2013.

18 Sichel JY, Dangoor E, Eliashar R and Halperin D: Management of congenital laryngeal malformations. Am J Otolaryngology 21: 22-30, 2000.

19 Mong A, Johnson AM, Kramer SS, Coleman BG, Hedrick HL, Kreiger P, Flake A, Johnson M, Wilson RD, Adzick NS and Jaramillo D: Congenital high airway obstruction syndrome: MR/US findings, effect on management, and outcome. Pediatr Radiol 38: 1171-1179, 2008.
Received May 2, 2017

Revised May 28, 2017

Accepted May 30, 2017 